\title{
Self-consistent stationary MHD shear flows in the solar atmosphere as electric field generators
}

\author{
D. H. Nickeler ${ }^{1}$, M. Karlický1 ${ }^{1}$ T. Wiegelmann ${ }^{2}$, and M. Kraus ${ }^{1}$ \\ 1 Astronomical Institute, AV ČR, Fričova 298, 25165 Ondřejov, Czech Republic \\ e-mail: dieter.nickeler@asu.cas.cz \\ 2 Max-Planck Institut für Sonnensystemforschung, Justus-von-Liebig-Weg 3, 37077 Göttingen, Germany
}

Received 16 March 2014 / Accepted 2 July 2014

\begin{abstract}
Context. Magnetic fields and flows in coronal structures, for example, in gradual phases in flares, can be described by 2D and 3D magnetohydrostatic (MHS) and steady magnetohydrodynamic (MHD) equilibria.

Aims. Within a physically simplified, but exact mathematical model, we study the electric currents and corresponding electric fields generated by shear flows.

Methods. Starting from exact and analytically calculated magnetic potential fields, we solved the nonlinear MHD equations selfconsistently. By applying a magnetic shear flow and assuming a nonideal MHD environment, we calculated an electric field via Faraday's law. The formal solution for the electromagnetic field allowed us to compute an expression of an effective resistivity similar to the collisionless Speiser resistivity.

Results. We find that the electric field can be highly spatially structured, or in other words, filamented. The electric field component parallel to the magnetic field is the dominant component and is high where the resistivity has a maximum. The electric field is a potential field, therefore, the highest energy gain of the particles can be directly derived from the corresponding voltage. In our example of a coronal post-flare scenario we obtain electron energies of tens of $\mathrm{keV}$, which are on the same order of magnitude as found observationally. This energy serves as a source for heating and acceleration of particles.
\end{abstract}

Key words. magnetohydrodynamics (MHD) - Sun: flares - Sun: corona - methods: analytical

\section{Introduction}

Dissipation or acceleration processes of energized particles occur in a variety of astrophysical plasma environments. For example, acceleration of charged particles is observed in the heliosphere, where anomalous cosmic rays are accelerated to high energies (see, e.g., Drake et al. 2010; Giacalone et al. 2012), in the Earth magnetotail and aurorae (e.g., Birn et al. 2012), and in solar flares (see, e.g., Miller 1998; Aschwanden 2002) and nanoflares (e.g., Bingert \& Peter 2011), where electrons and ions are heated and accelerated. These processes are typically connected with strong electric currents and electric fields. A reasonable approach for computing these electric fields and currents is provided by the theory of magnetohydrodynamics (MHD). But while MHD simulations compute solutions only on pre-defined grid points, meaning that values of the electromagnetic field have to be interpolated, analytical MHD configurations have the advantage of providing exact knowledge of the electromagnetic field at every point in space. Therefore, exact analytical MHD configurations are ideal as background fields in test particle simulations.

To trigger dissipation, for instance, in the form of Ohmic heating, or acceleration, a parallel component of the electric field with respect to the magnetic field must exist. Such electric field components parallel to the magnetic field can be obtained from nonideal MHD.

The heating of the solar plasma and the acceleration of charged particles during solar flare events is a long-standing problem. Three different main mechansisms have been described (for a detailed review see Aschwanden 2002): (i) DC-electric field acceleration, which is typically connected to magnetic collapse processes (magnetic reconnection such as collapsing magnetic loops and the magnetic mirror effect, Karlický \& Bárta 2007) or via the Betatron mechanism (Karlický \& Kosugi 2004), (ii) stochastic acceleration caused by wave-particle interaction, so-called weak turbulence (e.g., Miller 1998; Lazarian \& Vishniac 1999), and (iii) shock acceleration.

The scenario of DC-electric field acceleration is very promising, in particular for the aftermath of a solar flare event, where magnetic reconnection had taken place and a plasmoid was ejected. In such a reconnection region, strong electric fields are generated, which can directly accelerate charged particles. These particles are traced by their X-ray emission. However, after the new equilibrium state is reached, the main magnetic field component of such a post-flare configuration is the poloidal magnetic field, which can typically be described by a potential field. This can be justified by the fact that after the impulsive phase the main component of the field should be relaxed. However, the "bursty" reconnection event itself is not sufficient to explain the observed slow decay in intensity of the X-ray observations taken immediately after the impulsive phase (see, e.g., Kane 1974). This behavior of the emission speaks in favor of a continous (although reduced) acceleration on much longer timescales. If the relaxed configuration would consist of a pure potential field, this would imply that no further dissipation can take place and particle acceleration has stopped, in contrast to what is observed. Hence, particles must also be accelerated in the (almost) relaxed magnetic field. Therefore, a current-producing shear component must exist, which provides a reasonably strong electric field component that is necessary to accelerate charged particles along 
the field lines. Such shear fields have been observed (see, e.g., Wang 1992).

In this paper we investigate the influence of shear flows on the generation of electric fields with component parallel to the magnetic field in a typical post-flare configuration. In numerical test-particle approaches, as has been critically commented on by, e.g., Brown et al. (2009) and Zharkova et al. (2011), the particles are passive, which means that the feedback of the moving charges is not taken into account. This could be done numerically by considering a kinetic approach. However, because in kinetic models spatial and time scales have to be resolved, which requires quite different scales (Debye length and gyrofrequency), this treatment is numerically expensive. In contrast, our exact analytical nonideal MHD model allows us to precisely compute the field everywhere, not only on a predefined grid. In addition, our treatment of the accelerated bulk particles automatically includes the nonlinear feedback between the plasma and the electromagnetic field, which emphasizes the advantage of exact analytical models.

\section{Theoretical approach}

\subsection{Derivation of the MHD model}

In typical simulation scenarios a shear is applied to the footpoints of solar arcade structures (see, e.g., Leake et al. 2013, and references therein). Then the system relaxes into a new state. However, this new state is not necessarily an exact equilibrium state. Because we aim at an exact steady-state for our model considerations, we have to follow a strategy that allows us to compute the exact final state into which the system relaxes. This is offered by the transformation theory, which was developed by Gebhardt \& Kiessling (1992). The transformation method allows us to calculate steady ideal MHD equilibria with field-aligned incompressible flow from known MHS equilibria (see, e.g., Petrie \& Neukirch 1999; Nickeler et al. 2006, 2013; Nickeler \& Wiegelmann 2010, 2012), and it is applied here to obtain a stationary equilibrium, consisting of a poloidal field and a shear component in $z$-direction, from an originally pure potential field. Our chosen coordinate system is such that the $y$-axis is perpendicular to the solar surface (pointing upward), and the $x$-axis is tangential. The $z$-axis is tangential as well and points out of the (poloidal) plane in all our graphics.

We start from the set of stationary MHD equations for fieldaligned, incompressible flows, given by

$$
\begin{aligned}
\boldsymbol{\nabla} \cdot(\rho \boldsymbol{v}) & =0, \\
\rho(\boldsymbol{v} \cdot \boldsymbol{\nabla}) \boldsymbol{v} & =\boldsymbol{j} \times \boldsymbol{B}-\boldsymbol{\nabla} p, \\
\boldsymbol{\nabla} \times(\boldsymbol{v} \times \boldsymbol{B}) & =\mathbf{0}, \\
\boldsymbol{\nabla} \times \boldsymbol{B} & =\mu_{0} \boldsymbol{j}, \\
\boldsymbol{\nabla} \cdot \boldsymbol{B} & =0, \\
\boldsymbol{\nabla} \cdot \boldsymbol{v} & =0, \\
\boldsymbol{v} & = \pm\left|M_{\mathrm{A}}\right| \boldsymbol{v}_{\mathrm{A}} \\
\boldsymbol{v}_{\mathrm{A}} & :=\frac{\boldsymbol{B}}{\sqrt{\mu_{0} \rho}},
\end{aligned}
$$

where $\rho$ is the mass density, $\boldsymbol{v}$ is the plasma velocity, $\boldsymbol{B}$ is the magnetic flux density, $\boldsymbol{j}$ is the current density, $p$ is the plasma pressure, $M_{\mathrm{A}}$ is the Alfvén Mach number, $\boldsymbol{v}_{\mathrm{A}}$ is the Alfvén velocity, and $\mu_{0}$ is the magnetic permeability of the vacuum. The gravitational force in the considered domains of our model is at least a factor 100 lower than the Lorentz-force, so that the influence of gravity in our approach can be neglected.
In the current work we focus on solar magnetic arcade structures, implying translational invariance. This justifies restricting our investigations to $2.5 \mathrm{D}$ magnetic field configurations, that is, $\partial / \partial z=0$ for all our variables, although the transformation used could also be applied to full $3 \mathrm{D}$ scenarios. In addition to the translational invariance, we assume that there is no electric current component in the invariant (here $z$ ) direction, that is, $j_{z}=0$. As $j_{z}=0$, we have to solve the Laplace equation $\Delta A=0$ for the flux function $A$ or $\Delta \phi_{m}=0$ for the complex conjugated vector potential $\phi_{m}$. This is commonly achieved by complex analysis. Hence we define by $\phi_{m}$ and $A$ the complex conjugated potentials of the complex magnetic vector potential

$\mathcal{A}(u)=\phi_{m}+\mathrm{i} A$,

with $u=x+\mathrm{i} y$. These potentials fulfill the Cauchy-Riemann equations $\boldsymbol{\nabla} \phi_{m}=\boldsymbol{\nabla} A \times \boldsymbol{e}_{z}$ and, therefore, obey the condition $\boldsymbol{\nabla} \phi_{m}$. $\boldsymbol{\nabla} A=0$. To determine the magnetic potential $\phi_{m}$ and the flux function $A$, the Laplace equation is solved by expressing $\mathcal{A}(u)$ with Laurent series, applying asymptotical boundary conditions.

With the magnetic potentials, we can use the potential field $\boldsymbol{\nabla} \phi_{m}$ to define the static poloidal magnetic field $\boldsymbol{B}_{\mathrm{ps}}$ in the form

$\boldsymbol{B}_{\mathrm{ps}}=\sqrt{1-M_{\mathrm{A}}^{2}} \nabla \phi_{m}=\sqrt{1-M_{\mathrm{A}}^{2}} \boldsymbol{B}_{\mathrm{p}}$,

where $\boldsymbol{\nabla} \phi_{m}=\boldsymbol{B}_{\mathrm{p}}$ is the stationary poloidal magnetic field and $M_{\mathrm{A}}$ is the constant Alfvén Mach number. The requirement of $M_{\mathrm{A}}=$ const follows from the fact that on the one hand, $\boldsymbol{B}_{\mathrm{ps}}$ is a potential field, and on the other hand, no current is generated in $z$-direction by the transformation. For simplicity, to have the representation of the stationary magnetic field in the usual form via $\nabla \phi_{m}$, we introduced the factor $\sqrt{1-M_{\mathrm{A}}^{2}}$ into the static poloidal magnetic field, which vanishes identically after the transformation has been applied. For the current investigation, we considered only sub-Alfvénic flows, which means that $M_{\mathrm{A}}^{2}<1$. The initial potential magnetic field $\boldsymbol{B}_{\mathrm{ps}}$ together with the static plasma pressure $p_{\mathrm{s} 0}=$ const define the starting MHS equilibrium.

To compute the stationary MHD equilibrium, we applied a $2.5 \mathrm{D}$ shear flow $\boldsymbol{v}$ and simultaneously performed the transformation, so that we obtained a self-consistent 2.5D MHD flow. While the $x$ and $y$ components of the shear flow are functions of the poloidal magnetic field, the $z$ component produces a nonconstant magnetic shear $B_{z}=B_{z}(A)$ in $z$-direction, given by

$B_{z}(A)=\frac{v_{z}(A)}{M_{\mathrm{A}}} \sqrt{\mu_{0} \rho(A)}$,

where $\rho(A)$ is the plasma density. As was shown by Nickeler et al. (2006), the density is an explicit function of $A$. To fulfill the requirement of a field-aligned flow, the shear flow has to have the following structure

$\boldsymbol{v}=\frac{M_{\mathrm{A}}}{\sqrt{\mu_{0} \rho(A)\left(1-M_{\mathrm{A}}^{2}\right)}} \boldsymbol{B}_{\mathrm{ps}}+\frac{M_{\mathrm{A}}}{\sqrt{\mu_{0} \rho(A)}} B_{z}(A) \boldsymbol{e}_{z}$.

From the transformation and the application of the shear the stationary magnetic field has the form

$\boldsymbol{B}=\boldsymbol{B}_{\mathrm{p}}+B_{z}(A) \boldsymbol{e}_{z} \equiv \boldsymbol{\nabla} \phi_{m}+B_{z}(A) \boldsymbol{e}_{z}$,

and the electric current is given by Ampère's law,

$$
\begin{aligned}
\boldsymbol{j} & =\frac{1}{\mu_{0}} \boldsymbol{\nabla} \times B_{z} \boldsymbol{e}_{z}=\frac{1}{\mu_{0}} \boldsymbol{\nabla} B_{z} \times \boldsymbol{e}_{z} \\
& =\frac{1}{\mu_{0}} B_{z}^{\prime}(A) \boldsymbol{\nabla} A \times \boldsymbol{e}_{z}=\frac{1}{\mu_{0}} B_{z}^{\prime}(A) \nabla \phi_{m} .
\end{aligned}
$$


The prime denotes the derivative with respect to $A$, that is, $B_{z}^{\prime}(A)=\mathrm{d} B_{z} / \mathrm{d} A$. The thermal pressure of the sheared equilibrium is

$p=p_{\mathrm{s}}-\frac{\rho(A)}{2} \boldsymbol{v}^{2}=p_{\mathrm{s} 0}-\frac{1}{2 \mu_{0}} B_{z}^{2}(A)-\frac{M_{\mathrm{A}}^{2}}{2 \mu_{0}}|\nabla A|^{2}$.

The parameter $p_{\mathrm{s}}$ in Eq. (16) is the static pressure of an equilibrium state like ours plus a shear component. This means that we define the total static pressure as $p_{\mathrm{s}}=p_{\mathrm{s} 0}-\left(1-M_{\mathrm{A}}^{2}\right) B_{z}^{2} /\left(2 \mu_{0}\right)$, with $p_{\mathrm{s} 0}$ as the static pressure of the pure poloidal field. The thermal pressure (Eq. (16)) together with the flow (Eq. (12)) and the magnetic field (Eq. (13)) self-consistently fulfill the incompressible ideal steady-state MHD equations with field-aligned flows.

In ideal MHD, Ohm's law is given by

$\boldsymbol{E}+\boldsymbol{v} \times \boldsymbol{B}=\mathbf{0}$.

The use of field-aligned flows, which implies that $\boldsymbol{v} \times \boldsymbol{B}=\mathbf{0}$, has a severe consequence, because such an electro-magnetic field configuration in ideal MHD cannot accelerate injected charged particles because the electric field is zero. To guarantee particle acceleration, we must rely on resistive MHD, using resistive Ohm's law given by

$\boldsymbol{E}+\boldsymbol{v} \times \boldsymbol{B}=\eta \boldsymbol{j}$

In this scenario an electric field also exists for field-aligned flows, but only for $\eta \boldsymbol{j} \neq 0$ and hence in particular for $\eta \neq 0$.

To enable quasi-steady and sustainable electric fields with field-aligned flows, it is necessary to simultaneously solve the resistive Ohm's law and the momentum equation. This was first investigated by Grad \& Hogan (1970) and subsequently by Throumoulopoulos (1998) and Throumoulopoulos \& Tasso (2000) for the case of axisymmetric fields. These authors found several classes of analytical equilibria with physically plausible $\sigma=1 / \eta$ profiles, stating clearly that in general, fields with constant resistivity do not exist by proving that only the assumption of a spatially varying $\sigma$ makes the equilibrium problem well posed. In addition, they found that according to Ohm's law $\eta$ is determining the MHD solutions, but $\eta$ is also determined by constraints concerning the geometry of flow and field. Therefore, we applied a similar concept of a nonconstant resistivity for our translational invariant model.

The steady-state Maxwell equation $\boldsymbol{\nabla} \times \boldsymbol{E}=-\partial \boldsymbol{B} / \partial t=\mathbf{0}$ implies the existence of an electric potential $\phi_{\mathrm{e}}$ so that $-\boldsymbol{\nabla} \phi_{\mathrm{e}}=$ $\boldsymbol{E}=\eta \boldsymbol{j}$. Because the Cauchy-Rieman differential equations imply that $\boldsymbol{\nabla} \phi_{m} \cdot \boldsymbol{\nabla} A=0$, we can choose $\phi_{m}$ and $A$ as orthogonal coordinates. This allows us to consider the electric potential $\phi_{\mathrm{e}}$ as a function of the magnetic potential field $\phi_{m}$ and the flux function $A$. With the current, given by Eq. (15), we can re-write the identity $\eta \boldsymbol{j}=-\boldsymbol{\nabla} \phi_{\mathrm{e}}$ in the form

$\frac{1}{\mu_{0}} \eta\left(\phi_{m}, A\right) B_{z}^{\prime}(A) \nabla \phi_{m}=-\frac{\partial \phi_{\mathrm{e}}}{\partial \phi_{m}} \nabla \phi_{m}-\frac{\partial \phi_{\mathrm{e}}}{\partial A} \nabla A$.

A comparison of the coefficients delivers that $\partial \phi_{\mathrm{e}} / \partial A=0$, which means that the electric potential $\phi_{\mathrm{e}}$ is an explicit function of $\phi_{m}$ only, and therefore

$\frac{1}{\mu_{0}} \eta\left(\phi_{m}, A\right) B_{z}^{\prime}(A)=-\frac{\mathrm{d} \phi_{\mathrm{e}}\left(\phi_{m}\right)}{\mathrm{d} \phi_{m}}=: \frac{1}{\mu_{0}} \xi\left(\phi_{m}\right)$,

where $\xi\left(\phi_{m}\right)=\eta\left(\phi_{m}, A\right) B_{z}^{\prime}(A)$, which has the SI unit Ohm, can be regarded as the resistance of the plasma. Furthermore, we find from Eq. (20) for the electric potential $\phi_{\mathrm{e}}$

$\phi_{\mathrm{e}}=-\frac{1}{\mu_{0}} \int \xi\left(\phi_{m}\right) \mathrm{d} \phi_{m}$.
Consequently, equipotential surfaces of the scalar potential $\phi_{m}$ of the poloidal magnetic field are also equipotential surfaces of the electric potential, $\phi_{\mathrm{e}}=\phi_{\mathrm{e}}\left(\phi_{m}\right)$.

Considering the different representations, the electric field can be written in various equivalent forms:

$$
\begin{aligned}
\boldsymbol{E} & =\eta \boldsymbol{j}=\frac{\eta}{\mu_{0}} B_{z}^{\prime}(A) \boldsymbol{\nabla} \phi_{m}=\frac{1}{\mu_{0}} \xi\left(\phi_{m}\right) \boldsymbol{\nabla} \phi_{m} \\
& =\frac{1}{\mu_{0}} \boldsymbol{\nabla} \int \xi\left(\phi_{m}\right) \mathrm{d} \phi_{m}=-\boldsymbol{\nabla} \phi_{\mathrm{e}} .
\end{aligned}
$$

The existence of an electric field component aligned with the magnetic field provides an energy gain and hence an acceleration of charged particles along the field lines. The computation of the total electric field (and hence the parallel component) explicitly depends on the resistivity $\eta$, but implicitly on the resistance $\xi$. In the scenario of a post-flare configuration described above, which neglects currents in the invariant direction and an electric field component produced by the flow, we found that the resistivity is given by (see Eq. (20))

$\eta\left(\phi_{m}, A\right)=\frac{\xi\left(\phi_{m}\right)}{B_{z}^{\prime}(A)}$

Although the resistivity is an explicit function of the two complex conjugated potentials $\phi_{m}$ and $A$, it is very special in the sense that the two coordinates appear separately in the two functions $\xi$ and $B_{z}^{\prime}$ that determine $\eta$.

The resistance, $\xi$, and the derivative of the magnetic shear, $B_{z}^{\prime}$, can basically be chosen independently. To investigate the properties of this resistivity, we discuss various options for functions $\xi$ and $B_{z}^{\prime}$ in the following. The case of a constant magnetic shear component can directly be excluded because this would imply that $B_{z}^{\prime}(A)=0$ and hence the configuration would contain no currents and the resistivity would be undefined. For a nonconstant magnetic shear component we are left with four different possibilities: (i) $B_{z}^{\prime}(A)$ is constant and $\xi$ is constant; (ii) $B_{z}^{\prime}(A)$ is constant and $\xi$ is not constant; (iii) $B_{z}^{\prime}(A)$ is nonconstant but $\xi$ is constant; and (iv) $B_{z}^{\prime}(A)$ and $\xi$ are both nonconstant.

If $\xi$ and $B_{z}^{\prime}(A)$ were both constant, the resistivity would be constant as well, meaning that the electric field only depends on the poloidal magnetic field (see Eq. (22)). Hence, a strong electric field would only occur in regions of high magnetic field strength, for example, in regions of high current density (see Eq. (15)). Such regions of high current density occur close to the poles of multipolar regions, for instance. If $B_{z}^{\prime}(A)$ is constant and $\xi$ is not constant, $\eta$ only depends on $\phi_{m}$ and only varies in direction along the magnetic field lines. If $B_{z}^{\prime}(A) \neq$ constant, $\eta$ will decrease with increasing $B_{z}^{\prime}(A)$, but the choice of the function $\xi$ enables us to receive a high resistivity at specific locations. Hence choosing a constant $\xi$ is unsuitable because it prevents a change and localization of the electric field along the magnetic field lines and therefore an effective concentration of the particle acceleration engine.

The search for appropriate expressions for the resistivity is additionally hampered by the fact that in cases of nonconstant $\xi$ one has to find a physical explanation for $\xi$, which is not obvious a priori. Evidently, $\xi$ represents a characteristic electric resistance, which depends on the distance along the magnetic field lines.

A high resistivity alone is not sufficient for the occurrence of strong (parallel) electric fields. Instead, appropriate choices for the spatial distributions of both the poloidal magnetic field and the function $\xi$ are required. The regions where the conditions for the appearance of strong electric fields are met are, therefore, 
not necessarily identical to those where the effective resistivity $\eta$ is particularly high. Nevertheless, we need a physical explanation for the resistivity and a reasonable physical correlation between resistivity and the function $\xi$. The Spitzer resistivity is not valid for solar corona or solar flare scenarios because the density is too low, which prevents efficient collisions between particles. On the other hand, the turbulent collisionless resistivity (anomalous resistivity due to wave-particle interactions), which occurs during eruptive procsses such as impulsive phases of flares, is usually not stationary. Therefore, the most appropriate approach for our investigations is to find a stationary resistivity that takes noncollisional effects into account.

\subsection{Geometrical approach for the noncollisional inertia-induced resistivity: Speiser-like approach}

The resistivity in our model depends on the resistance $\xi$, which was defined in Eq. (20) as a function of the vector potential $\phi_{m}$. The resistance $\xi\left(\phi_{m}\right)$ and the resistivity $\eta=\xi\left(\phi_{m}\right) / B_{z}^{\prime}(A)$ define formally exact solutions for the resistivity and the electric field in the specific geometry of a given magnetic field, but for estimating the "amplitude", that is, the absolute value of $\xi$ and thus $\eta$, a quantitative approach is required. To estimate a noncollisional but steady-state $\eta$ it is hence essential to derive a parameterization for the resistance. For this, we make a short excursion into the two-fluid approach. Here, the resistance $\xi$ can be represented by utilizing the ideas of the so-called Speiser conductivity (Speiser 1970), which is based on the inertia of the injected and accelerated charged particles. This means that we are using stationary movements of particles to derive the relation between the total electric fields (see Eqs. (25) and (26) below) and $\xi$. This procedure can be justified by the suggestion of Speiser (see, e.g., Speiser 1968, 1970; Dungey \& Speiser 1969), who stated that the effect of particle inertia can be more important in determining the relation between $\boldsymbol{E}$ and $\boldsymbol{j}$ in a collisionless plasma than wave-particle interactions.

Differently from the situation in the Speiser models, the geometry used in our models is more complicated: our models are 2.5D while the Speiser models were 1.5D (see e.g. Lyons \& Speiser 1985). In addition, our current sheet is not given by the Maxwellian jump condition with respect to the main component $B_{\mathrm{p}}$ of the magnetic field, but by the shear component $B_{z}$.

In our model we assume that a steady-state flow of coronal material can develop in which the plasma streams along the field lines that have been sheared in z-direction. However, within this ordered plasma stream, a drift between the different species of charged particles (electrons and protons or ions) can be expected. The drift inside the global plasma flow initiates a current, which is, via Ampère's law, related to a magnetic shear component. Hence, no turbulent approach is needed to obtain an electric field. Instead, it results naturally from the inertial forces.

The inertial forces acting on the charged particles generated by the electric and magnetic fields can be written as

$$
\begin{aligned}
& \boldsymbol{E}+\boldsymbol{v}_{\mathrm{i}} \times \boldsymbol{B}=\frac{m_{\mathrm{i}} \boldsymbol{a}_{\mathrm{i}}}{q}:=\boldsymbol{E}_{\mathrm{i}}, \\
& \boldsymbol{E}+\boldsymbol{v}_{\mathrm{e}} \times \boldsymbol{B}=-\frac{m_{\mathrm{e}} \boldsymbol{a}_{\mathrm{e}}}{e}:=\boldsymbol{E}_{\mathrm{e}},
\end{aligned}
$$

where $\boldsymbol{v}_{\mathrm{i}}$ and $\boldsymbol{v}_{\mathrm{e}}$ are the velocity fields of the charged particles (ions $\mathrm{i}$ and electrons e), $m_{\mathrm{i}}$ and $m_{\mathrm{e}}$ are their masses, $\boldsymbol{a}_{\mathrm{i}}$ and $\boldsymbol{a}_{\mathrm{e}}$ are the accelerations acting on the bulk of particles, and $q$ and $e$ are their charges. The coupling between the Maxwell equation (Ampère's law) and the fluid equations is realized via Eqs. (30), (31) and (33) below. Here, we explicitly considered only the electric force and the Lorentz force, while all other collective forces (like $\boldsymbol{\nabla} P_{\mathrm{e}}$, the Hall term, etc.) are included in the total electric field for each species (ions $\boldsymbol{E}_{\mathrm{i}}$ and electrons $\boldsymbol{E}_{\mathrm{e}}$ ). The forces $\boldsymbol{F}_{\mathrm{i}, \mathrm{e}}=m_{\mathrm{i}, \mathrm{e}} \boldsymbol{a}_{\mathrm{i}, \mathrm{e}}$ are proportional to the total electric fields $E_{\mathrm{i}, \mathrm{e}}(=$ the electric field that particles encounter in the comoving frame). If we solve for the velocity fields $\boldsymbol{v}_{\mathrm{i}, \mathrm{e}}$, the general solution of the force Eqs. (25) and (26) is

$$
\begin{aligned}
& \boldsymbol{v}_{\mathrm{i}}=\frac{\left(\boldsymbol{E}-\boldsymbol{E}_{\mathrm{i}}\right) \times \boldsymbol{B}}{|\boldsymbol{B}|^{2}}+\lambda_{\mathrm{i}} \boldsymbol{B}, \\
& \boldsymbol{v}_{\mathrm{e}}=\frac{\left(\boldsymbol{E}-\boldsymbol{E}_{\mathrm{e}}\right) \times \boldsymbol{B}}{|\boldsymbol{B}|^{2}}+\lambda_{\mathrm{e}} \boldsymbol{B} .
\end{aligned}
$$

The free parameters $\lambda_{\mathrm{i}, \mathrm{e}}$ of the general solution correspond to the Alfvén Mach number of the flows of the particular particle species:

$M_{\mathrm{i}}^{2}:=\lambda_{\mathrm{i}}^{2} \mu_{0} n_{\mathrm{i}} q, \quad$ and $\quad M_{\mathrm{e}}^{2}:=\lambda_{\mathrm{e}}^{2} \mu_{0} n_{\mathrm{e}} e$.

The electric current is typically given by the drift between the different charges

$\boldsymbol{j}:=n_{\mathrm{i}} q \boldsymbol{v}_{\mathrm{i}}-n_{\mathrm{e}} e \boldsymbol{v}_{\mathrm{e}}$.

On the other hand, in the MHD picture the current is generated by the magnetic shear (see Eq. (15))

$\boldsymbol{j}=\frac{1}{\mu_{0}} B_{z}^{\prime}(A) \nabla \phi_{m}$.

As both expressions have to be equal, we can compare the coefficients. For this, we introduce a new orthogonal coordinate system defined by the basis $\left((\nabla A)^{0}, \boldsymbol{e}_{z},\left(\nabla \phi_{m}\right)^{0}\right)$. The superscript 0 denotes that the vectors are normalized. Then we can express the physical parameters in this new coordinate system: The poloidal magnetic field can be written as $\boldsymbol{B}_{\mathrm{p}}=B_{\mathrm{p}}\left(\boldsymbol{\nabla} \phi_{m}\right)^{0}$, and the total electric fields that are needed to compute the particle velocities become

$\boldsymbol{E}_{\mathrm{i}, \mathrm{e}}=\alpha_{\mathrm{i}, \mathrm{e}}(\boldsymbol{\nabla} A)^{0}+\beta_{\mathrm{i}, \mathrm{e}} \boldsymbol{e}_{z}+\gamma_{\mathrm{i}, \mathrm{e}}\left(\boldsymbol{\nabla} \phi_{m}\right)^{0}$,

where $\alpha, \beta$, and $\gamma$ are the coordinates of the corresponding basis. The relation for the current density can thus be written in the form

$\boldsymbol{j}:=n_{\mathrm{i}} q \boldsymbol{v}_{i}-n_{\mathrm{e}} e \boldsymbol{v}_{\mathrm{e}} \stackrel{!}{=} \frac{1}{\mu_{0}} B_{z}^{\prime} B_{\mathrm{p}}\left(\boldsymbol{\nabla} \phi_{m}\right)^{0}$.

The expressions for the velocities, Eqs. (27) and (28), and a comparison of coefficients with respect to the orthogonal unit vectors result in three conditions for the electric field components of the two species:

$$
\begin{aligned}
j_{\mathrm{A}}= & \frac{n_{\mathrm{e}} e-n_{\mathrm{i}} q}{\mu_{0}|\boldsymbol{B}|^{2}} \xi B_{z} B_{\mathrm{p}}-\frac{n_{\mathrm{i}} q \beta_{\mathrm{i}} B_{\mathrm{p}}}{|\boldsymbol{B}|^{2}}+\frac{n_{\mathrm{i}} q \gamma_{\mathrm{i}} B_{z}}{|\boldsymbol{B}|^{2}} \\
& +\frac{n_{\mathrm{e}} e \beta_{\mathrm{e}} B_{\mathrm{p}}}{|\boldsymbol{B}|^{2}}-\frac{n_{\mathrm{e}} e \gamma_{\mathrm{e}} B_{z}}{|\boldsymbol{B}|^{2}} \\
j_{z}= & \frac{n_{\mathrm{i}} q \alpha_{\mathrm{i}} B_{\mathrm{p}}}{|\boldsymbol{B}|^{2}}-\frac{n_{\mathrm{e}} q \alpha_{\mathrm{e}} B_{\mathrm{p}}}{|\boldsymbol{B}|^{2}}+\left(n_{\mathrm{i}} q \lambda_{\mathrm{i}}-n_{\mathrm{e}} e \lambda_{\mathrm{e}}\right) B_{z} \\
j_{\phi_{m}}= & -\frac{n_{\mathrm{i}} q \alpha_{\mathrm{i}} B_{z}}{|\boldsymbol{B}|^{2}}+\frac{n_{\mathrm{e}} e \alpha_{\mathrm{e}} B_{z}}{|\boldsymbol{B}|^{2}}+\left(n_{\mathrm{i}} q \lambda_{\mathrm{i}}-n_{\mathrm{e}} e \lambda_{\mathrm{e}}\right) B_{\mathrm{p}}
\end{aligned}
$$

with the projection in the direction of the three coordinates of the three basis vectors, namely

$$
\begin{aligned}
j_{A} & :=\boldsymbol{j} \cdot(\boldsymbol{\nabla} A)^{0}=0, \\
j_{z} & :=\boldsymbol{j} \cdot \boldsymbol{e}_{z}=0, \\
j_{\phi_{m}} & :=\boldsymbol{j} \cdot\left(\boldsymbol{\nabla} \phi_{m}\right)^{0}=\frac{1}{\mu_{0}} B_{z}^{\prime} B_{\mathrm{p}} .
\end{aligned}
$$


Because only $j_{A}$ contains the resistance $\xi$, we concentrate on this component to receive a relation coupling the physical parameters $B_{\mathrm{p}}, B_{z}, \xi$, and $\eta$. This coupling can only be calculated if $n_{\mathrm{e}} e-$ $n_{\mathrm{i}} q$ in the nominator of the first term on the right-hand side of Eq. (34) does not vanish. This condition is only fullfilled as long as $n_{\mathrm{i}} \neq n_{\mathrm{e}}$ with $n_{\mathrm{i}} \approx n_{\mathrm{e}}$.

We define the following conductivities:

$\sigma_{\mathrm{g}, \mathrm{e}}=\frac{n_{\mathrm{e}} e}{|\boldsymbol{B}|} \quad$ and $\quad \sigma_{\mathrm{g}, \mathrm{i}}=\frac{n_{\mathrm{i}} q}{|\boldsymbol{B}|}$

which resemble the gyroconductivity introduced by Speiser (1968). However, in the definition of Speiser's gyroconductivity only the $B_{z}$ component was used. This component was oriented perpendicular to the antiparallel field lines of the magnetic neutral sheet, so that particles within the neutral sheet are gyrating around $B_{z}$ and therefore encounter an electric field (in their own frame of reference, see Lyons \& Speiser 1985). Since, in our case, there exists no magnetic neutral sheet, we have to use $|\boldsymbol{B}|=\sqrt{B_{\mathrm{p}}^{2}+B_{z}^{2}}$ for the corresponding magnetic field, and the particles gyrate around its field lines.

By inserting the definition of the gyroconductivities (Eq. (40)) into the equation of the current (Eq. (34)), we obtain

$0=\left(\sigma_{\mathrm{g}, \mathrm{e}}-\sigma_{\mathrm{g}, \mathrm{i}}\right) \frac{\xi B_{\mathrm{p}}}{\mu_{0}} \frac{B_{z}}{|\boldsymbol{B}|}+\frac{\sigma_{\mathrm{g}, \mathrm{i}}}{|\boldsymbol{B}|}\left(B_{z} \gamma_{\mathrm{i}}-B_{\mathrm{p}} \beta_{\mathrm{i}}\right)+\frac{\sigma_{\mathrm{g}, \mathrm{e}}}{|\boldsymbol{B}|}\left(B_{\mathrm{p}} \beta_{\mathrm{i}}-B_{z} \gamma_{\mathrm{i}}\right)$,

from which $\xi$ can in principle be calculated. In the situation in which $\beta_{\mathrm{i}}=\beta_{\mathrm{e}} \neq \gamma_{\mathrm{i}}=\gamma_{\mathrm{e}}$, the resistance $\xi$ results in

$\xi=\mu_{0} \frac{B_{z} \gamma_{\mathrm{i}}-B_{\mathrm{p}} \beta_{\mathrm{i}}}{B_{\mathrm{p}} B_{z}}$

This scenario is very unlikely, as it implies that the electrons (owing to their much smaller mass) would run away from the ions because the electric field accelerates the lighter electrons much more strongly than the heavy ions. Hence, the configuration would not reach a stationary state.

For the ions to achieve about the same acceleration as the electrons, the total electric field encountered by the electrons must be negligible compared with the total field encountered by the ions. Therefore, if we set in Eq. (41) $\beta_{\mathrm{i}} \gg \beta_{\mathrm{e}}$ and $\gamma_{\mathrm{i}} \gg \gamma_{\mathrm{e}}$, which is physically more reasonable, we obtain the following expression for $\xi$ :

$\xi=\mu_{0} \frac{\sigma_{\mathrm{g}, \mathrm{i}}}{\sigma_{\mathrm{g}, \mathrm{e}}-\sigma_{\mathrm{g}, \mathrm{i}}} \frac{B_{z} \gamma_{\mathrm{i}}-B_{\mathrm{p}} \beta_{\mathrm{i}}}{B_{z} B_{\mathrm{p}}}$.

With the help of this formula we can estimate the Speiser-like resistivity.

To ensure that the poloidal component of $\boldsymbol{E}_{i}$ is the dominating one, we demand that

$\gamma_{i} \approx\left|\boldsymbol{E}_{\mathrm{i}}\right|=\eta_{\mathrm{i}}|\boldsymbol{j}|=\frac{\eta_{\mathrm{i}}}{\mu_{0}} \frac{\mathrm{d} B_{z}(A)}{\mathrm{d} A} B_{\mathrm{p}}$,

where the term on the right-hand side again refers to the current of the MHD picture. Then, Eq. (44) can be rewritten in the form

$\frac{\gamma_{\mathrm{i}}}{B_{\mathrm{p}}}=\frac{1}{\mu_{0} \sigma_{\mathrm{g}, \mathrm{i}}} \frac{\mathrm{d} B_{z}(A)}{\mathrm{d} A}$

where we used the identity for the resistivity $\eta_{\mathrm{i}} \equiv \frac{1}{\sigma_{\mathrm{g}, \mathrm{i}}}$. Because we have no precise information about the acceleration terms from MHD theory, it is physically reasonable to request that the acceleration should be field-aligned, that is,

$\frac{\gamma_{\mathrm{i}}}{B_{\mathrm{p}}} \approx \frac{\beta_{i}}{B_{z}}$

We now define

$\frac{\beta_{\mathrm{i}} B_{\mathrm{p}}}{\gamma_{\mathrm{i}} B_{z}}:=\varepsilon_{1} \approx 1$.

Therefore, $\xi$ can be written as

$$
\begin{aligned}
\xi & =\mu_{0} \frac{\sigma_{\mathrm{g}, \mathrm{i}}}{\sigma_{\mathrm{g}, \mathrm{e}}\left(1-\frac{\sigma_{\mathrm{g}, \mathrm{i}}}{\sigma_{\mathrm{g}, \mathrm{e}}}\right)} \frac{\gamma_{\mathrm{i}}}{B_{\mathrm{p}}}\left(1-\varepsilon_{1}\right) \\
& =\mu_{0} \frac{\sigma_{\mathrm{g}, \mathrm{i}}}{\sigma_{\mathrm{g}, \mathrm{e}}\left(1-\varepsilon_{2}\right)} \frac{\gamma_{\mathrm{i}}}{B_{\mathrm{p}}}\left(1-\varepsilon_{1}\right) \\
& =\mu_{0} \frac{\sigma_{\mathrm{g}, \mathrm{i}}}{\sigma_{\mathrm{g}, \mathrm{e}}\left(1-\varepsilon_{2}\right)} \frac{B_{z}^{\prime}(A)}{\mu_{0} \sigma_{\mathrm{g}, \mathrm{i}}}\left(1-\varepsilon_{1}\right)=\frac{1-\varepsilon_{1}}{1-\varepsilon_{2}} \frac{B_{z}^{\prime}(A)}{\sigma_{\mathrm{g}, \mathrm{e}}},
\end{aligned}
$$

where we introduced the parameter $\varepsilon_{2}=\sigma_{\mathrm{g}, \mathrm{i}} / \sigma_{\mathrm{g}, \mathrm{e}}$. Because according to Eq. (24) $\xi=\eta B_{z}^{\prime}(A)$, the resistivity has the form

$\eta=\frac{1-\varepsilon_{1}}{1-\varepsilon_{2}} \frac{1}{\sigma_{\mathrm{g}, \mathrm{e}}}=: \varepsilon \frac{1}{\sigma_{\mathrm{g}, \mathrm{e}}}$.

Owing to the quasi-neutrality $\varepsilon_{2} \approx 1$, which means that $\eta \sim$ $1 / \sigma_{\mathrm{g}, \mathrm{e}}=\eta_{\mathrm{e}}$. Consequently, the total resistivity, and therefore also the function $\xi$, is related to the gyroresistivity of the electrons.

The resistivity (hence the Ohmic heating) depends on the chosen geometry and on the constraint of only having almost field-aligned forces. To keep the resistivity finite, a deviation from exact neutrality (=quasi-neutrality) is required. To keep this deviation small, the field-aligned forces have to be chosen appropriately, so that in the expression of the resistivity, Eq. (51), the "geometrical" term $\left(1-\varepsilon_{1}\right)$, which describes the deviation from field-aligned acceleration, compensates for the denominator $\left(1-\varepsilon_{2}\right)$, which describes the deviation from perfect neutrality. This compensation should be made in such a way that $\eta$ is bounded, implying that $\varepsilon$ is on the order of unity or at least bounded by some finite value. This guarantees that the resistivity $\eta$ can be computed at all. A second important criterion why $\varepsilon$ should be bounded and on the order of unity comes from calculating the energies of the charged particles. Because according to Eq. (21) the electric potential results from integrating over the resistance $\xi$, the magnitude of the voltage that the electric particles can achieve is largely determined by the value of $\varepsilon$. If $\varepsilon$ were much higher than unity, the energies of the particles would approach the relativistic regime, in which the theory is not valid anymore.

In the following section we present one example with representative physical parameters and simplified magnetic field configuration.

\section{Results}

The scenario under investigation assumes that the field is almost relaxed, which supports the assumption of a potential field for the poloidal component of the field (here: the $x$ and $y$ components in the translational invariant magnetic field configuration). We concentrate on a small region around the footpoint of a solar arcade structure with dimensions $3 \mathrm{Mm} \times 3 \mathrm{Mm}$. This domain was chosen to avoid bipolar magnetic field structures with high 


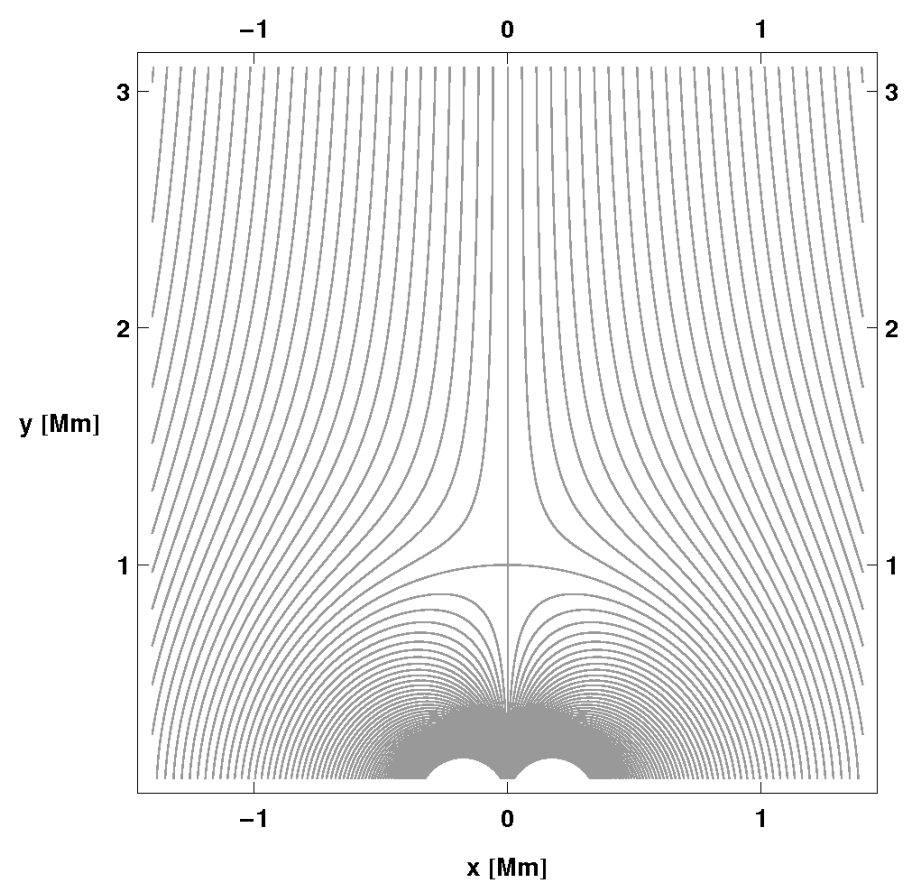

Fig. 1. Field line projection onto the poloidal plane.

plasma $\beta$ that contain current sheets with a component of the current in $z$-direction. We represent the magnetic field configuration with a $2 \mathrm{D}$ dipole superimposed on a homogeneous, monopolar field component in $y$-direction. This results in a dome-like structure at the bottom and an X-point separatrix on top. This configuration is achieved by expanding the complex magnetic vector potential, $\mathcal{A}$, in a Laurent series of the form

$\mathcal{A}=\sum_{k=-\infty}^{k=\infty} C_{k} u^{k}=-\mathrm{i} B_{\infty} u+\frac{C}{u}$,

with the complex constants $C_{k}$ of which only those for the homogeneous component $\left(C_{1}=-\mathrm{i} B_{\infty}\right)$ and the dipole component $\left(C_{-1}=\mathrm{i}|C|\right)$ are considered, while all others are set to zero. The constant $|C|$ in the latter is given by $|C|=B_{\infty} R^{2}$, where $R$ corresponds to the height $y$ above the dipole at which the poloidal field vanishes. This height marks the location of the magnetic null point. The choice of the constants guarantees that the asymptotical boundary condition, namely $\boldsymbol{B}(|u| \rightarrow \infty)=B_{\infty} \boldsymbol{e}_{y}$, is fulfilled. The field lines of the configuration are displayed in Fig. 1. Because this figure shows the projection of the field lines onto the poloidal plane, it represents the two cases before and after the magnetic shear is applied that results from the shear flow (see Eq. (11)). To compute these field lines, we chose values appropriate for the solar corona. Magnetic field determinations are usually complicated and values obtained from observations at different locations and wavelength regions range from about $10^{-3} \mathrm{~T}$ to $10^{-1} \mathrm{~T}$ (see, e.g., Lin et al. 2000; Brosius \& White 2006; Caspi \& Lin 2010). We adopted a mean value of the magnetic field of $B_{\infty}=10^{-2} \mathrm{~T}$ and for the height $R=1 \mathrm{Mm}$. The contour lines of the magnetic potential $\phi_{m}$ are depicted in Fig. 2. They are everywhere perpendicular to the magnetic field lines.

The only effective electric field component that can act as a particle accelerator is the component parallel to the magnetic field. From the representations of the total magnetic field (Eq. (13)) and the electric field (Eq. (23)), the parallel

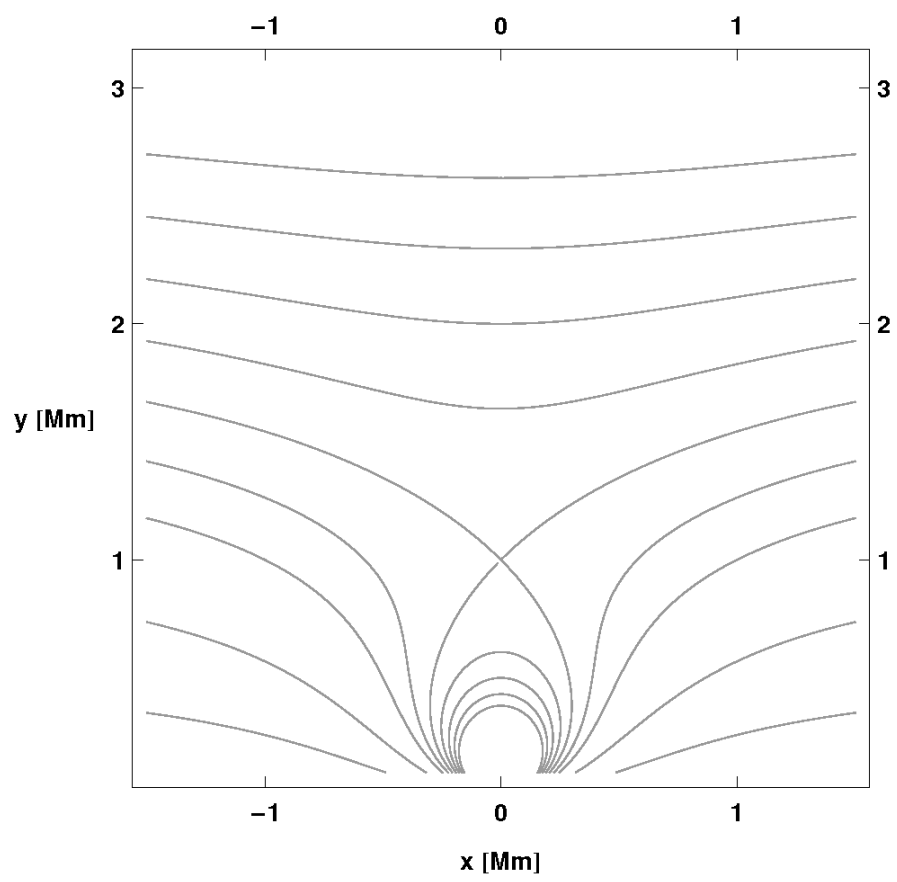

Fig. 2. Contour lines of the magnetic potential.

component of the electric field, $E_{\|}$, results in

$E_{\|}=\frac{\boldsymbol{E} \cdot \boldsymbol{B}}{|\boldsymbol{B}|}=\frac{1}{\mu_{0}} \xi\left(\phi_{m}\right) \frac{\left|\boldsymbol{\nabla} \phi_{m}\right|}{\sqrt{1+B_{z}^{2}(A) /\left|\boldsymbol{\nabla} \phi_{m}\right|^{2}}}$.

To maximize $E_{\|}$, it is essential to choose a rather small magnetic shear component ${ }^{1}$. However, the magnetic shear should neither be constant nor zero, because this would imply a current-free field.

The crucial term in computing $E_{\|}$is the resistance function $\xi$. This function was defined in Eq. (20) to be a pure funtion of $\phi_{m}$. However, as was shown in Eq. (50), it contains the derivative of the magnetic shear component, which is a pure function of $A$. Hence, we need to find a reasonable approach for $B_{z}^{\prime}(A)$, that allows us to determine the profile of the function $\xi\left(\phi_{m}\right)$. According to Eq. (50), $\xi$ is given by

$\xi\left(\phi_{m}\right)=\varepsilon \frac{|\boldsymbol{B}|}{n_{\mathrm{e}} e} B_{z}^{\prime}(A)$.

Because we request that the null point of the poloidal magnetic field is also a null point of the complete magnetic field, $B_{z}(A=0)$ has to vanish. A Taylor expansion of $B_{z}(A)$ around the null point hence delivers that in the lowest order of $A$ the magnetic shear component must have the form

$B_{z}(A)=$ const $\cdot A$,

because the contour line $A=0$ is the separatrix. The shape of the magnetic shear component $B_{z}(A)$ is shown in Fig. 3. For the computation we chose a value for the constant in Eq. (55) of

1 The $B_{z}$ component is not really restricted to low values as every $B_{z}=B_{z}(A)$ produces an equilibrium because of the noncanonical transformation method. But low values of the shear component compared with the main poloidal component of the field are of advantage to maximize $E_{\|}$, to justify the assumption of quasi-neutrality, and to fulfill the dominance of the poloidal over the $z$-component of the total electric field of the ions (see Eq. (47)). 


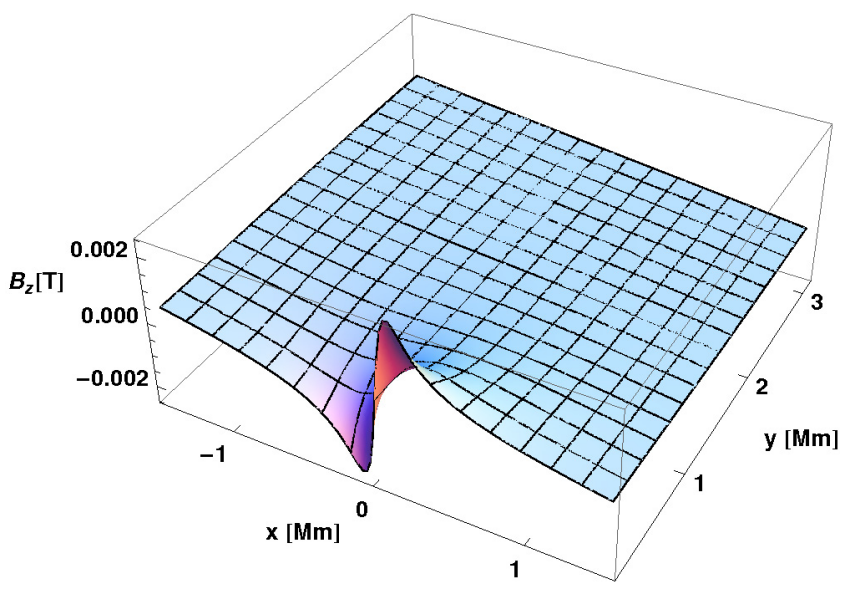

Fig. 3. Spatial distribution of the magnetic shear component.

$5 \times 10^{-8} \mathrm{~m}^{-1}$ to guarantee that $\left|B_{z}\right|<\left|B_{\mathrm{p}}\right|$. Given the highest values of $\sim 10^{-3} \mathrm{~T}$ in the dipole region, the magnetic shear component is still very small compared with the superimposed poloidal field $\left(B_{\infty} \sim 10^{-2} \mathrm{~T}\right)$.

The absolute value of the electric current over the considered domain and the corresponding contour plot are shown in Fig. 4, while the individual contributions in $x$ and $y$ direction are shown in Fig. 5. The current flows along the poloidal field direction, that is, in $x$ - and $y$-direction. Above the dipole region, here for $y>1 \mathrm{Mm}$, the total current is very low and approaches a constant value. This is visible in the top right panel of Fig. 4, which shows the total current at large distances, and also from the contour plots, which show the increasing separation of the contour lines with increasing distance from the dipole. In the region of the dipole field, the $x$-component of the current flows into the positive $x$-direction in the left part of the dome, and oppositely in the right part. The dominant component of the current is the $y$ component, which diverges close to the pole.

The region around the null point can be "evacuated", for example, after an ejection of a flux rope. This means that the total magnetic field and the electron density both approach zero. Outside the null point region, where the magnetic field saturates, we assume that the density saturates as well. Therefore, the term $|\boldsymbol{B}| /\left(n_{\mathrm{e}} e\right)=\left|\boldsymbol{B}_{\infty}\right| /\left(n_{\mathrm{e}, \infty} e\right)$ can be assumed to be approximately constant. The chosen values for $B_{\infty}$ and $n_{\mathrm{e}, \infty}$ can be adjusted to typical coronal values. We emphasize, however, that not every arbitrary combination of $|\boldsymbol{B}|$ and $n_{\mathrm{e}}$ will successfully deliver a strong enough resistivity or electric field. The last unknown in the function $\xi$, which we define as the profile function $\xi_{0}\left(\phi_{m}\right)$, is the term $\varepsilon$. This term is requested to cover the $\phi_{m}$ dependence of the resistance $\xi$. For our test calculations we set $\left|\xi_{0}\left(\phi_{m}\right)\right| \leq 1$ to guarantee the quasi-neutrality condition and the physical significance, in other words, to keep the $\varepsilon$-term bounded as physical requirement.

For different choices of $B_{z}^{\prime}(A)$ and $\boldsymbol{B}_{\mathrm{p}}$, we must also recognize that the magnetic shear has to be chosen such that $|\boldsymbol{B}| B_{z}^{\prime}(A) /($ ne) results in a reasonable value for the amplitude of the energy per charge unit, the voltage $\phi_{\mathrm{e}}\left(\phi_{m}\right)$. Otherwise the $\varepsilon$-term has to be adjusted, which changes the constraint $\left|\xi_{0}\left(\phi_{m}\right)\right| \leq 1$. The implication of a lower value of $B_{\mathrm{p}}$ and/or a higher value of the density has then to be compensated for either by an enhancement of $B_{z}^{\prime}(A)$ or an increase of $\varepsilon$, which causes enhanced deviation from neutrality relative to the deviation from the field-aligned acceleration. This would not cause problems, because the deviation from neutrality will usually be extremly small, such that any relative change or fluctuation of the $\varepsilon_{1}$-parameter will be larger. For one single profile function with $B_{z}^{\prime}(A)=1 / l_{0}$, where $l_{0}$ is a typical lengthscale for the shear that we set to $2 \times 10^{7} \mathrm{~m}$, we can write

$\eta=\varepsilon \frac{l_{0}}{\sigma_{\mathrm{ge}}}=\xi_{0}\left(\phi_{m}\right) l_{0}\left\langle\frac{1}{\sigma_{\mathrm{ge}}}\right\rangle$.

However, our approach allows defining multiple sites for acceleration and heating via a fragmented resistivity,

$$
\eta=\sum_{i} \frac{\xi_{0, i} l_{0}}{\left\langle\sigma_{\mathrm{ge}}\right\rangle_{i}}
$$

The sign of the individual $\xi_{0, i}$ can be either positive or negative, and accordingly, the direction of acceleration can change at each of these multiple acceleration sites.

In the current investigation, we focused on an example using one single, "monolithic" profile of the form

$\xi_{0}=\cosh ^{-2}\left(\frac{\phi_{m}-21500 \mathrm{Tm}}{1000 \mathrm{Tm}}\right)$

From observations (e.g., Aschwanden \& Benz 1997), electron densities in acceleration sites of solar flares of $(0.6-10) \times 10^{15} \mathrm{~m}^{-3}$ were measured. As acceleration regions are usually regions where the electron density is preferentially low (see, e.g., Aschwanden 2002), we fixed it in our model at $n_{\mathrm{e}, \infty}=10^{14} \mathrm{~m}^{-3}$. Figure 6 shows the resulting resistivity, $\eta$, in the computed domain, which for our chosen simplifications is directly proportional to the total resistance $\xi$. The resistivity shows a kinked wall-like structure above the null point region and a ring-shaped wall below. This is easily understood from inspecting Eq. (54) and the plot of the contour lines of the magnetic potential (Fig. 2). Because the resistivity is basically the same function as the profile function $\xi_{0}$, which itself is only a function of the magnetic potential $\phi_{m}$ while all other terms in Eq. (54) are constant, the resistivity reaches a maximum where $\xi_{0}$ has a maximum. As the isocontours of the function $\phi_{m}$ have two disjoint branches at the numerical value of $\phi_{m}=21500 \mathrm{Tm}$, every global function of $\phi_{m}$ also has two disjoint isocountours with the same isocontour value.

The resulting spatial variation of $E_{\|}$is shown in Fig. 7. This parallel component of the electric field is almost identical to the total electric field (see Fig. 8). Particles are strongest affected, that is, heated and accelerated, in the domains in which $E_{\|}$is high. These are also the regions of highest voltage, as is obvious from Fig. 9. Because according to the mathematical frame of our theory we are able to superimpose different $\xi_{0}$ profiles, it is possible to construct fragmented structures of multiple walls, which provide many regions of enhanced electric field that are suitable for consecutive heating (and acceleration) of the particles.

\section{Discussion and conclusions}

A parallel electric field component tends to accelerate particles, especially electrons, out of the thermal distribution, resulting in so-called runaway particles. However, the acceleration only works efficiently if the parallel electric field component is sufficiently high. Otherwise, the energy gained from the electric field will mainly be dissipated into heat by dynamical friction (in a collisionless plasma as in our case) or collisions (in a collisional plasma). Such dissipation connected with Ohmic heating 

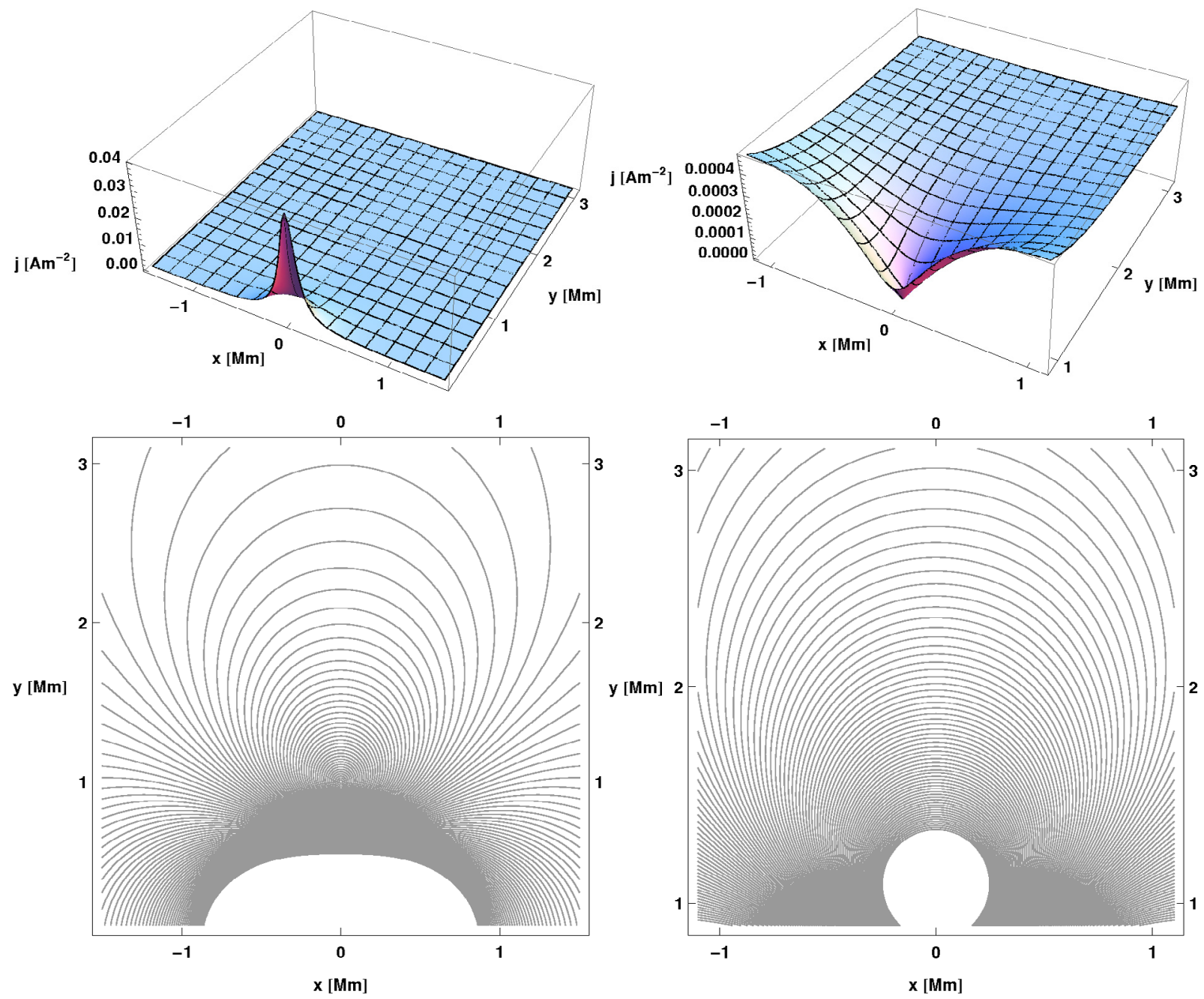

Fig. 4. Total current of the stationary state (top) and its contour lines (bottom). For better visualization the panels to the right display the behavior of the current beyond the dipole region, i.e. at far distances.
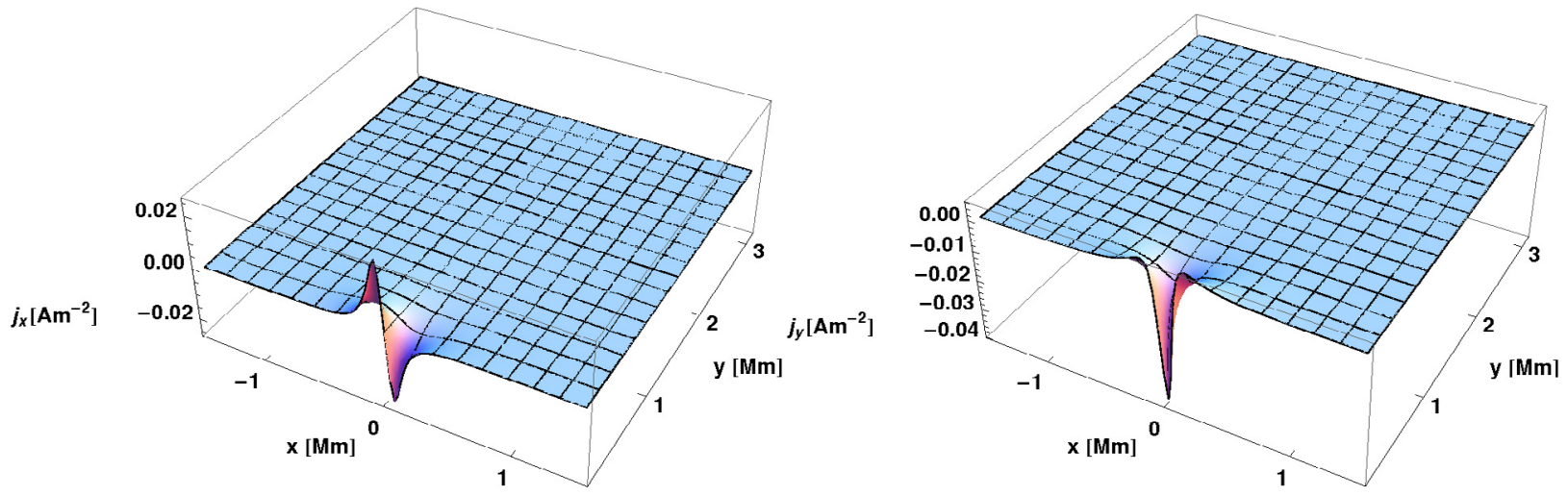

Fig. 5. Electric current of the stationary state. Displayed are the $x$-component (left) and the $y$-component (right).

has been shown to be a reliable mechanism for coronal heating based on detailed 3D numerical MHD simulations (see, e.g., Bingert \& Peter 2011; Bourdin et al. 2013).

To measure the effectivity of particle acceleration, the parallel electric field strength $E_{\|}$is typically compared with the Dreicer electric field $E_{\mathrm{D}}$ (Dreicer 1960). This Dreicer field is clearly defined in collisional plasmas, where it is on the order of $E_{\mathrm{D}} \simeq 6 \times 10^{-4}\left(n_{14} T_{6}^{-1}\right) \mathrm{V} \mathrm{m}^{-1}$, where $n_{14}$ is the plasma density in units of $10^{14} \mathrm{~m}^{-3}$, and $T_{6}$ is the temperature in units of $10^{6} \mathrm{~K}$. For collisionless plasmas, the definition of the Dreicer field is less straightforward and has only been considered for the anomalous resistivity, where the effective collision frequency is defined, and was found to be 4 to 6 orders of magnitude higher than the classical Spitzer value (e.g., Papadopoulos 1977; Priest \& Forbes 2000).

In our collisionless scenario with Speiser-like (particle inertia) resistivity, a proper definition of a corresponding Dreicer field fails. Because of the nonlinearity between the electric field 


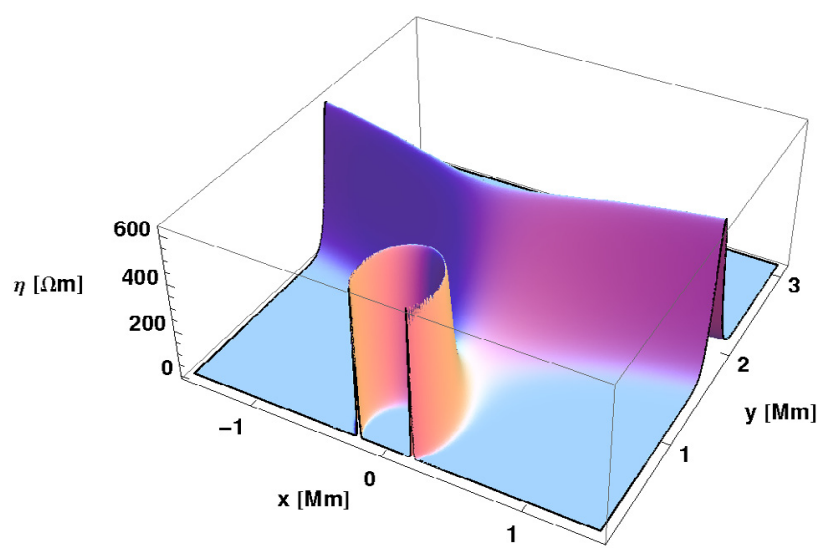

Fig. 6. Spatial distribution of the resistivity $\eta$.

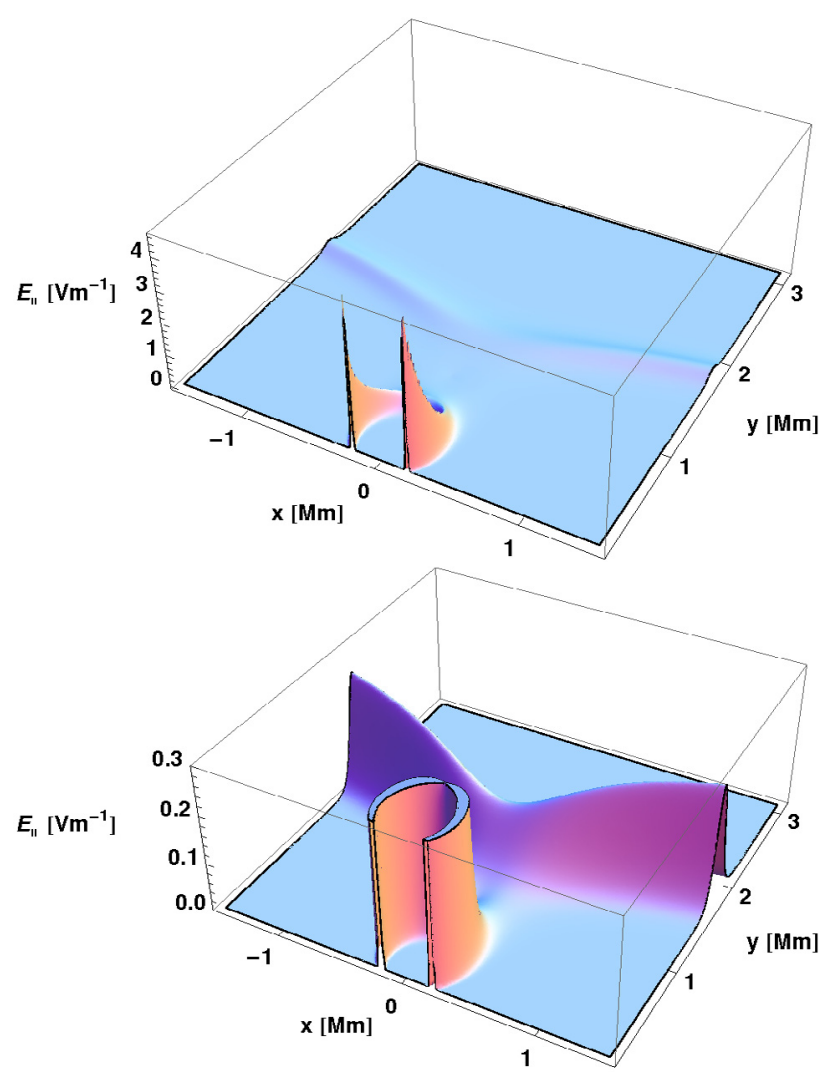

Fig. 7. Parallel electric field component. For better visualization, the bottom panel shows $E_{\|}$cut off at a numerical value of $0.3 \mathrm{~V} / \mathrm{m}$.

and the current, no effective collision frequency can be computed. Instead, there exists a nonlinear interplay between the particle movement and their relative drift and the geometrical structure of the electromagnetic field. Therefore the resistivity does not depend linearly on a collision frequency (as is the case for the Spitzer and the anomalous or turbulent resistivity), so that the effective Dreicer field $E_{\mathrm{D}_{*}}$ cannot simply be calculated via the relation $E_{\mathrm{D}_{*}}=\left(\eta_{*} / \eta_{0}\right) E_{\mathrm{D}}$ given by Norman $\&$ Smith (1978): In the classical view, the current density $j=E_{\mathrm{D}} / \eta_{0}$ is fixed, so that any enhancement of $\eta_{0}$ causes an increase of the Dreicer field. The inertia-based resistivity approach does not allow fixing the current density and simultaneously enlarging the resistivity without enlarging magnetic field and/or reducing parameterically the density. This is caused by the definition of the differential electric potential $\xi$. The relation obtained by Norman $\&$ Smith (1978) assumes that the change of the resistivity has

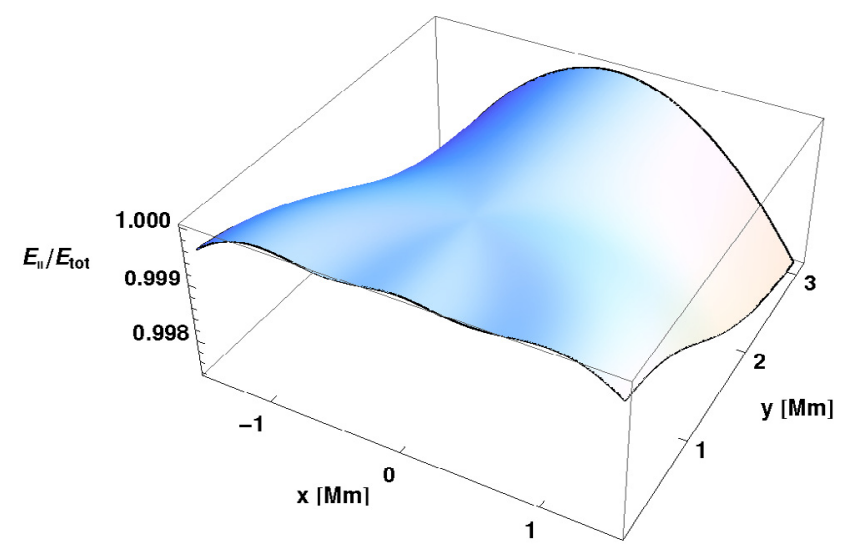

Fig. 8. Ratio of the parallel and total electric fields.

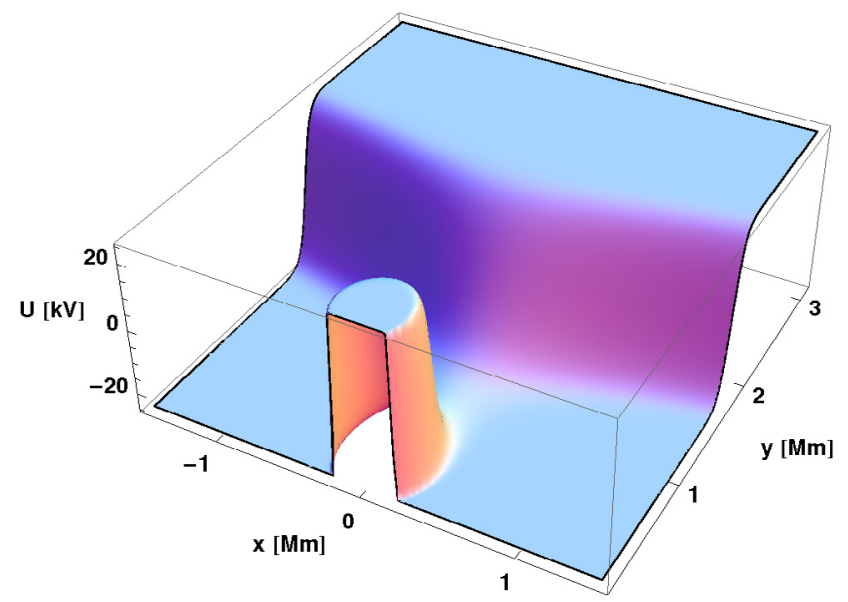

Fig. 9. Spatial distribution of the voltage.

no influence on the current density. This is not the case in our scenario where, in general, the resistivity and the current density depend parametrically on the differential magnetic shear. Furthermore, Eq. (24) implies that raising the current by raising the differential magnetic shear $B_{z}^{\prime}(A)$ alone, the noncollisional resistivity even decreases. The assumption that the current is (approximately) fixed implies that $j \propto B_{z}^{\prime} B_{\mathrm{p}}=$ constant $^{2}$. Every parametrical increase of $B_{z}^{\prime}$ and $B_{z}$ leads to a decrease of $B_{\mathrm{p}}$ and therefore also of the electric field, because $|\boldsymbol{E}| \propto \xi B_{\mathrm{p}}$. Thus $\xi \propto \sqrt{1+\left(B_{z} / B_{\mathrm{p}}\right)^{2}} B_{z}^{\prime} B_{\mathrm{p}}$ leads to a slight enhancement (if $\left|B_{z} / B_{\mathrm{p}}\right| \lesssim 1$ ) of $\xi$, but the price is the total decrease of the electric field. Increasing $B_{\mathrm{p}}$ alone would lead to an increase of $\eta$ and of the electric field.

One might doubt the role of the $\varepsilon_{i}$-terms, of course, but they mainly depend on a geometrical factor, where the deviation from quasi-neutrality must correspond to the deviation from fieldaligned acceleration to avoid the decoupling of $\xi$ from the twospecies system and guarantee the regularity of $\xi$ (bounded value for the electric field and $\xi$ ). This term can in principle change the Speiser-like resistivity by order(s) of magnitude, but, as it must be bounded and has to compensate for the smallness of the deviation from field-aligned acceleration, it only has a marginal influence in our approach.

Hence we have no clear diagnostics at hand to estimate the efficiency of particle acceleration. However, according to our

\footnotetext{
2 As $B_{\mathrm{p}}$ is a function of $\phi_{m}$ and $A$ in general, it is not possible to keep the current fixed.
} 
initial conditions and requirements (quasi-neutrality, low drift velocity), no strong acceleration of the bulk particles is expected. Instead, only particles in the high-energy tail of the Maxwellian distribution might be affected because for them even a (very) small fraction of the classical Dreicer electric field is sufficient to accelerate them into runaway particles. However, as the resulting electric field in our model is almost completely parallel to the magnetic field, the particles will experience some acceleration along the field lines. Our whole scenario is based on a slight charge separation and a separate treatment of ions and electrons. An ultimate investigation of these processes requires a proper two-fluid analysis.

Although a full two-fluid analysis is beyond the scope of the current investigation, we can use the two-fluid perspective and the parameters from our model calculations to compute averaged velocities of the particles in the straight field-line region ${ }^{3}$ and estimate from this the highest and lowest energy of the bulk particles in our plasma model. The electric current density in the straight field-line region is approximately $3 \times 10^{-4} \mathrm{~A} \mathrm{~m}^{-2}$ (see Fig. 4). On the other hand, the current density in the two-fluid picture is connected to the particle velocities via $j=n_{\mathrm{i}} q v_{\mathrm{i}}-n_{\mathrm{e}} e v_{\mathrm{e}}$. We assume that the ion velocity should be on the order of the bulk velocity, which is $v_{\mathrm{i}} \approx M_{\mathrm{A}} B / \sqrt{\mu_{0} \rho}$, where $B \approx 10^{-2} \mathrm{~T}$, and $\rho \approx n_{\mathrm{i}} m_{p}$. Furthermore, the absolute values of the charges of the electron and ion (i.e., in our case protons) are equal, and the electron and ion densities are approximately equal because of quasineutrality and have (in our model) a value of $n_{\mathrm{i}} \approx n_{\mathrm{e}}=10^{14} \mathrm{~m}^{-3}$. This results in an electron velocity of $v_{\mathrm{e}} \approx 1 / 3 M_{\mathrm{A}} \times 10^{8} \mathrm{~m} \mathrm{~s}^{-1}$. If we assume a minimum Alfvén Mach number of 0.1 and a maximum of $\lesssim 1$, the energy of the bulk electrons is between about $1 \mathrm{keV}$ and $10 \mathrm{keV}$. In contrast, typical coronal values of the thermal energy of electrons for temperatures in the range $10^{6} \mathrm{~K}$ to $10^{7} \mathrm{~K}$ are about $0.1 \mathrm{keV}$ or $1 \mathrm{keV}$.

Based on our sheared potential field model, we achieve voltage values $(\sim 10 \mathrm{kV})$ in agreement with observed X-ray emission from solar flares (e.g., with RHESSI, see Aschwanden 2002; Önel \& Mann 2009). This voltage value can be up to an order of magnitude higher if we allow for a higher value of $B_{z}^{\prime}(A)$. However, the highest voltage is not high enough to produce the highly energetic particles with energies in $\mathrm{MeV}$ and even $\mathrm{GeV}$ range (see, e.g., Hurford et al. 2003). Our calculated example basically produces a single wall or sheet (see Fig. 7), meaning that particles are practically accelerated only once. For a sustained acceleration of the bulk, multiple walls are necessary. In the frame of our anlysis, a high number of consecutive walls, even with different amplitudes, can be obtained if we allow that either $B_{z}(A)$ or the differential electric potential $\xi$ or both are functions with a high spatial variation (fragmented). Under such considerations the voltage will also be fragmented, producing numerous solitary-wave-shaped "walls". The existence of such multiple fractal structures within the electromagnetic field allows repetetive acceleration (or decelaration) processes to very high energies as well. However, for a spatially variable magnetic shear component the $\phi_{m}$ dependence of the resistance $\xi$ cannot be expressed in a simple way, and the null point of the initial poloidal potential field is not necessarily conserved anymore. The pure linear dependence of $B_{z}(A)$ on $A$ considered in the presented example is a severe restriction. Better models in which $B_{z}(A)$ can be adjusted to constraints and boundary conditions require that in general $\sigma_{\mathrm{g}, \mathrm{e}}$ must also explicitly depend on $A$ and $\phi_{m}$, which complicates the analysis. Furthermore, for a more consistent investigation the generalized Ohm's law needs to be considered, and for this the MHD should be replaced by a real two-fluid approach.

Nevertheless, the great advantage of our MHD model is that it explicitly identifies the current caused by the drift between the accelerated particles with the current caused by the magnetic shear component. It is thus a valuable and self-consistent approach in the frame of nonideal MHD, which automatically incorporates the nonlinear electromagnetic feedback of the particles, which is ignored in the usual test particle approach.

Acknowledgements. We thank the anonymous referee for useful comments and suggestions on the draft. This research made use of the NASA Astrophysics Data System (ADS). D.H.N. and M.K. acknowledge financial support from GA ČR under grant numbers 13-24782 and P209/12/0103, respectively. The Astronomical Institute Ondřejov is supported by the project RVO:67985815.

\section{References}

Aschwanden, M. J. 2002, Space Sci. Rev., 101, 1

Aschwanden, M. J., \& Benz, A. O. 1997, ApJ, 480, 825

Bingert, S., \& Peter, H. 2011, A\&A, 530, A112

Birn, J., Artemyev, A. V., Baker, D. N., et al. 2012, Space Sci. Rev., 173, 49

Bourdin, P.-A., Bingert, S., \& Peter, H. 2013, A\&A, 555, A123

Brosius, J. W., \& White, S. M. 2006, ApJ, 641, L69

Brown, J. C., Turkmani, R., Kontar, E. P., MacKinnon, A. L., \& Vlahos, L. 2009, A\&A, 508, 993

Caspi, A., \& Lin, R. P. 2010, ApJ, 725, L161

Drake, J. F., Opher, M., Swisdak, M., \& Chamoun, J. N. 2010, ApJ, 709, 963

Dreicer, H. 1960, Phys. Rev., 117, 329

Dungey, J. W., \& Speiser, T. W. 1969, Planet. Space Sci., 17, 1285

Gebhardt, U., \& Kiessling, M. 1992, Phys. Fluids B, 4, 1689

Giacalone, J., Drake, J. F., \& Jokipii, J. R. 2012, Space Sci. Rev., 173, 283

Grad, H., \& Hogan, J. 1970, Phys. Rev. Lett., 24, 1337

Hurford, G. J., Schwartz, R. A., Krucker, S., et al. 2003, ApJ, 595, L77

Kane, S. R. 1974, in Coronal Disturbances, ed. G. A. Newkirk, IAU Symp., 57, 105

Karlický, M., \& Bárta, M. 2007, Adv. Space Res., 39, 1427

Karlický, M., \& Kosugi, T. 2004, A\&A, 419, 1159

Lazarian, A., \& Vishniac, E. T. 1999, ApJ, 517, 700

Leake, J. E., Linton, M. G., \& Török, T. 2013, ApJ, 778, 99

Lin, H., Penn, M. J., \& Tomczyk, S. 2000, ApJ, 541, L83

Lyons, L. R., \& Speiser, T. W. 1985, J. Geophys. Res., 90, 8543

Miller, J. A. 1998, Space Sci. Rev., 86, 79

Nickeler, D. H., \& Wiegelmann, T. 2010, Ann. Geophys., 28, 1523

Nickeler, D. H., \& Wiegelmann, T. 2012, Ann. Geophys., 30, 545

Nickeler, D. H., Goedbloed, J. P., \& Fahr, H.-J. 2006, A\&A, 454, 797

Nickeler, D. H., Karlický, M., Wiegelmann, T., \& Kraus, M. 2013, A\&A, 556, A61

Norman, C. A., \& Smith, R. A. 1978, A\&A, 68, 145

Önel, H., \& Mann, G. 2009, Central Eur. Astrophys. Bull., 33, 141

Papadopoulos, K. 1977, Rev. Geophys. Space Phys., 15, 113

Petrie, G. J. D., \& Neukirch, T. 1999, Geophys. Astrophys. Fluid Dyn., 91, 269

Priest, E., \& Forbes, T. 2000, Magnetic Reconnection

Speiser, T. W. 1968, in Earth's Particles and Fields, ed. B. M. McCormac, 393

Speiser, T. W. 1970, Planet. Space Sci., 18, 613

Throumoulopoulos, G. N. 1998, J. Plasma Phys., 59, 303

Throumoulopoulos, G. N., \& Tasso, H. 2000, J. Plasma Phys., 64, 601

Wang, H. 1992, Sol. Phys., 140, 85

Zharkova, V. V., Arzner, K., Benz, A. O., et al. 2011, Space Sci. Rev., 159, 357

3 The region, where the asymptotic boundary condition is reached. 